\title{
The common rate control account of prediction motion
}

\author{
Alexis D. J. Makin ${ }^{1}$ \\ Published online: 1 December 2017 \\ (C) The Author(s) 2017. This article is an open access publication
}

even when not driving or playing sports. After all, temporary occlusions often result from blinks or visually disruptive changes in viewpoint.

In the lab, occluded motion processing has been studied with prediction-motion (PM) tasks (see Fig. 1). PM tasks have a long history (Gottsdanker, 1956; Slater-Hammel, 1955; Wiener, 1962) and have sometimes been called motion extrapolation or time-to-contact tasks (with terminology partly reflecting the author's theoretical assumptions). Modern PM experiments typically require the participant to press a button when the occluded moving target arrives at a goal (Battaglini, Campana, \& Casco, 2013; Baurès, Oberfeld, \& Hecht, 2010, 2011; Benguigui, Broderick, \& Ripoll, 2004; DeLucia, Tresilian, \& Meyer, 2000; Makin, Poliakoff, Chen, \& Stewart, 2008; Makin, Stewart, \& Poliakoff, 2009; Peterken, Brown, \& Bowman, 1991; Rosenbaum, 1975; Sokolov \& Pavlova, 2003). This type of PM task is sometimes called a 'production task' because participants produce a motor response (see Fig. 1a). Another variant requires the participant to judge whether the target reappeared from occlusion too early or too late (Bennett \& Benguigui, 2013; DeLucia \& Liddell, 1998; Jonikaitis, Deubel, \& de'Sperati, 2009; Lyon \& Waag, 1995; O'Reilly, Mesulam, \& Nobre, 2008). This is sometimes called an 'interruption paradigm' (see Fig. 1b) because the target trajectory is interrupted. Production tasks and interruption paradigms are both types of PM task.

The first eye-tracking study with occluded moving targets was published 87 years ago (Travis \& Dodge, 1930), and eye tracking across occlusion has now been examined in detail (e.g. Becker \& Fuchs, 1985; Bennett \& Barnes, 2003; Churchland, Chou, \& Lisberger, 2003; Makin \& Poliakoff, 2011; Pola \& Wyatt, 1997). Meanwhile, developmental psychologists have used eye tracking to investigate the emergence of PM abilities in infants (von Hofsten, Kochukhova, \& Rosander, 2007). 
A) Production task

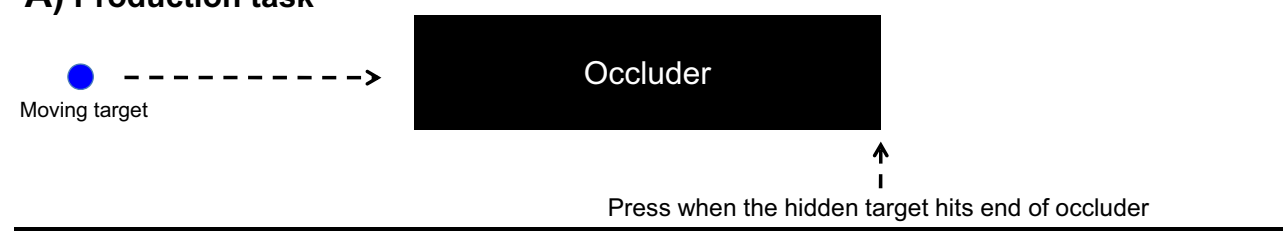

B) Interruption paradigm

Press when the hidden target hits end of occluder

O- - - - - - -

C) Arrival time estimation with approach motion

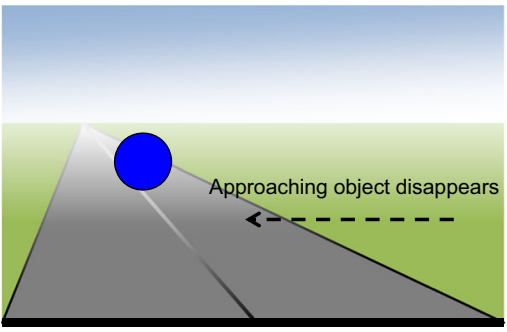

Press when the hidden object would hit you!
Reappearance too early or too late?

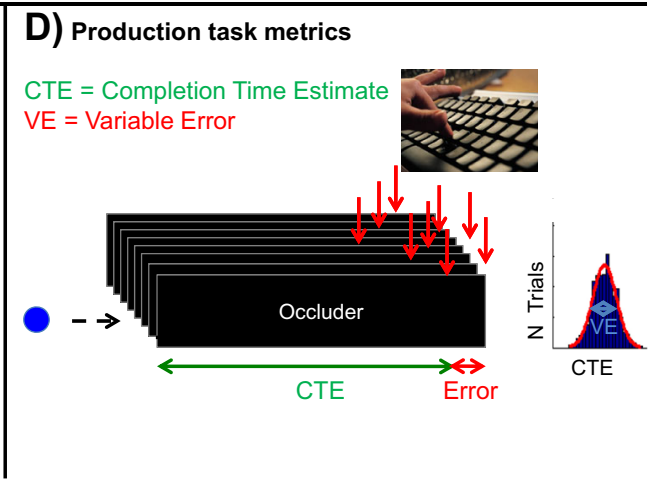

Variable error (VE) is the standard deviation of CTEs across repeated trials in a condition. (Colour figure online)

mechanisms (top down). The brain must run on its own during occlusion, without feedback from the senses.

Some researchers have used PM as a way of tapping the cognitive processes they seek to understand. PM can indeed be a useful probe; however, there is a risk of making underanalysed assumptions about the cognitive systems recruited during PM tasks. Supplementary Materials 1 Section 1 reviews three PM papers to illustrate the risk (Gilden, Blake, \& Hurst, 1995; Roth, Synofzik, \& Lindner, 2013; Vagnoni, Lourenco, \& Longo, 2012).

Hopefully, the current synthesis will allow future researchers to acquaint themselves with the range of possible mechanisms that might mediate PM. I have reviewed as much PM literature as possible, but, most importantly, I also introduce the new common rate control model.

\section{Using the supplementary materials}

To make the review more digestible, I have shifted a lot of detail to four Supplementary Materials sections. These are NOT essential reading. Supplementary Materials 1 provides extra commentary and literature review. Supplementary Materials 2 explains PM performance metrics and analysis. Supplementary Materials 3 and 4 are the detailed methods and results sections of Experiments 1 and 2. Code and data from the experiments can be found on Open Science Framework (osf.io/g4zm7). 


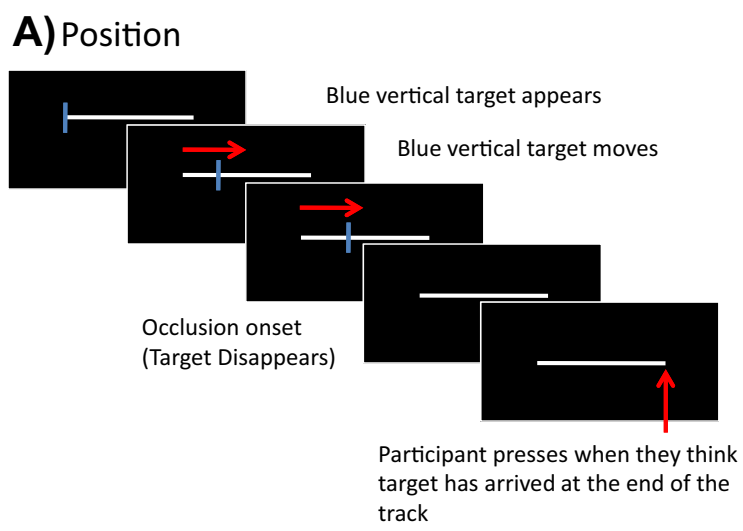

\section{B) Number}

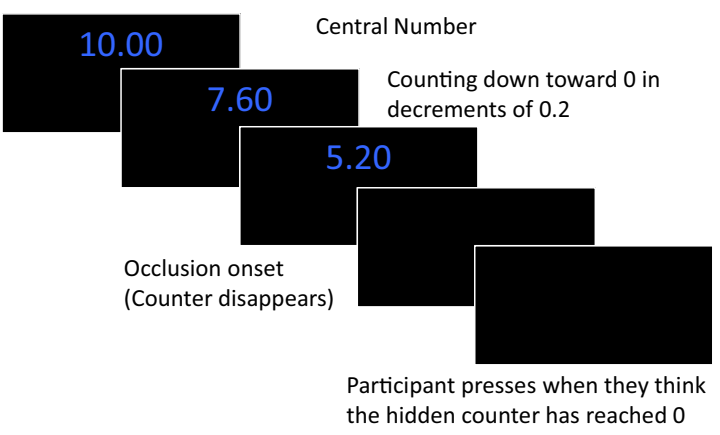

D) Accumulation

C) Colour

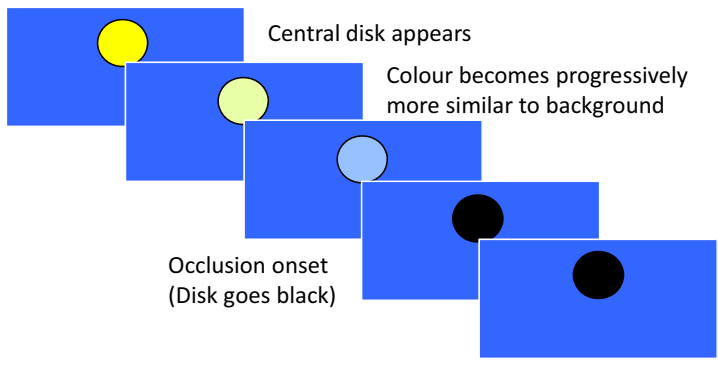

Participant presses when they think hidden colour matches the

background colour

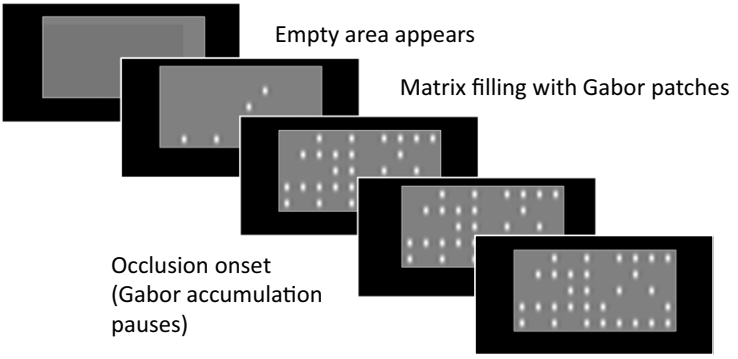

Participant presses when they think the matrix of gabors is completely full

Fig. 2 PM tasks in different dimensions. In all examples, there is a dynamic process on the screen, which becomes occluded. Participants assume continuation of the dynamic process and then press when they judge it to be complete. (Colour figure online)

\section{A common rate controller or many separate rate controllers?}

An object's position is only one variable that can visibly change over time. We are also sensitive to feature motion, such as change in number space or colour space (Blaser, Pylyshyn, \& Holcombe, 2000; Blaser \& Sperling, 2008; Sheth, Nijhawan, \& Shimojo, 2000).

Makin and Bertamini (2014) and Makin and Chauhan (2014) compared performance in different kinds of production task, including a standard position task (see Fig. 2a), and novel production tasks with occluded motion through feature space (see Fig. 2b-d). In all tasks, participants may run a dynamic mental simulation of the occluded process. Participants would then press the button when their mental simulation matched an indicated goal state. If this story is correct, PM tasks must involve a rate control mechanism that ensures such mental simulations are updated at the right speed (not too fast, not too slow).

There could be a common rate controller for updating in different dimensions (e.g. position, number, colour, accumulation). This is called the common rate control (CRC) hypothesis. Alternatively, there might be separate rate controllers for updating in each dimension. This is called the separate rate control (SRC) hypothesis. Figure $3 \mathrm{a}$ and $3 \mathrm{~b}$ show cartoon versions of the competing CRC and SRC alternatives (Makin, 2017).

Makin and Bertamini (2014) reasoned that if two PM tasks employ a common rate controller then performance should be similar in both, but if they employ separate rate controllers performance could be different. This line of reasoning hits an immediate barrier: How similar should performance be for CRC, and how different for SRC? It is difficult to decide upon a principled criterion for adjudicating between the competing models.

One partial solution is to focus on a metric that taps the functioning of the rate controller. Completion time estimates (CTEs) and variable error (VE, the standard deviation of CTEs) are linearly related to occlusion duration (see Fig. 3cd). If separate rate controllers with different characteristics are used in each task, then any task differences will grow with occlusion duration, resulting in different slopes. However, if the same rate controller is used in both tasks, then slopes should be comparable (even if intercepts differ). Makin and Bertamini (2014) and Makin and Chauhan (2014) found that slopes were often comparable in pairs of PM tasks and thus argued in favour of the CRC (Supplementary Materials 2 describes slope analysis in detail).

Lyon and Waag (1995) also found that performance accuracy declined with occlusion duration. Their data could be 

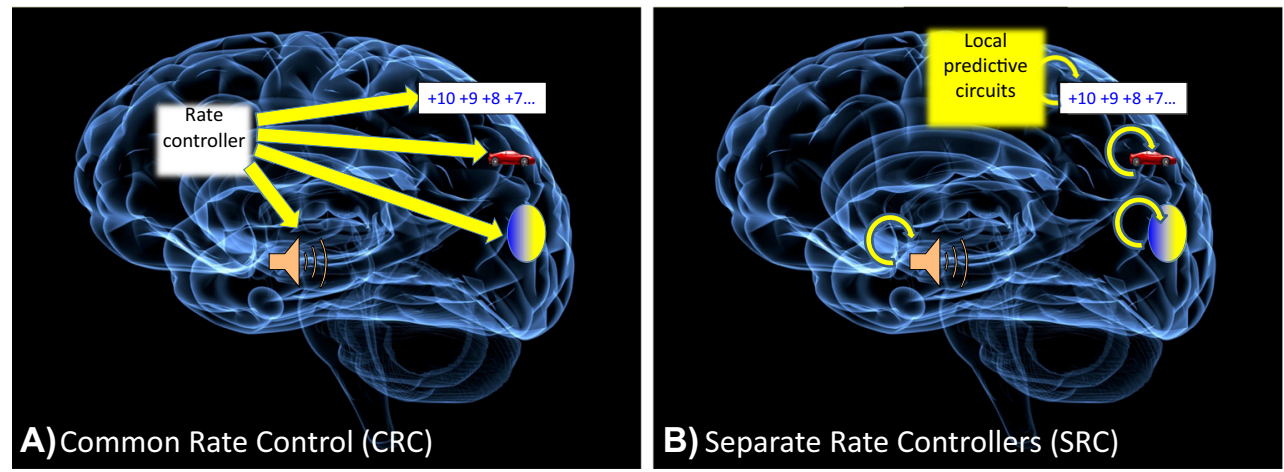

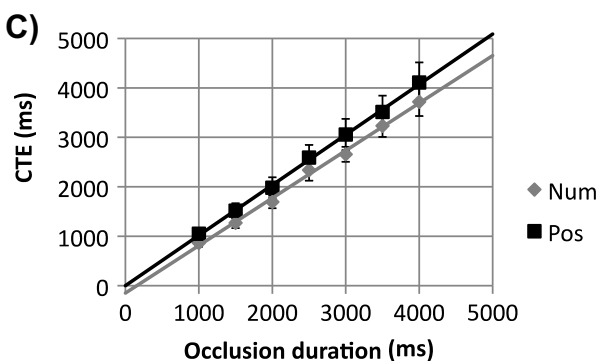

Fig. 3 Cartoon depictions of the common rate control (CRC) model (a) and the separate rate control (SRC) models (b). c CTE versus occlusion duration in position and number PM tasks. d VE versus occlusion duration in position and number PM tasks. Note how the slope of the

modelled with computer simulations that included random variations in 'tracker velocity' between trials (steady tracker) or moment-by-moment variations within a trial (unsteady tracker). We can now extend this claim: Decline in accuracy with occlusion duration arises from random variations in mental simulation velocity, which partly arises from noise in rate control systems (rather than just variations in tracker velocity).

So, according to the rationale of Makin and Bertamini (2014), intertask slope similarities support the CRC model. But is this rationale correct? What if separate PM tasks recruit separate rate control mechanisms, but all these rate control mechanisms had very similar properties?

It is unlikely that every conceivable PM task has a dedicated rate control module all to itself-that reading of the SRC is a straw man. However, all sensory systems could be endowed with ubiquitous microcircuits that do the routine job of making predictions about subsequent inputs (Alink, Schwiedrzik, Kohler, Singer, \& Muckli, 2010). Maybe PM performance is uniform across feature spaces because prediction is implemented in a uniform way across sensory maps? Performance on all different PM tasks could be comparable by accident rather than by design. This is fundamentally different from the CRC hypothesis, which proposes that a dedicated, anatomically discrete rate control module must be temporarily coupled to sensory maps during occlusion (see Fig. 3a).

Retinotopic visual areas are tiled with elementary motion detectors (EMDs). EMDs have small receptive fields tuned to velocity (Burr \& Thompson, 2011). Watamaniuk, McKee, and Grzywacz (1995) concluded that trajectory detection across

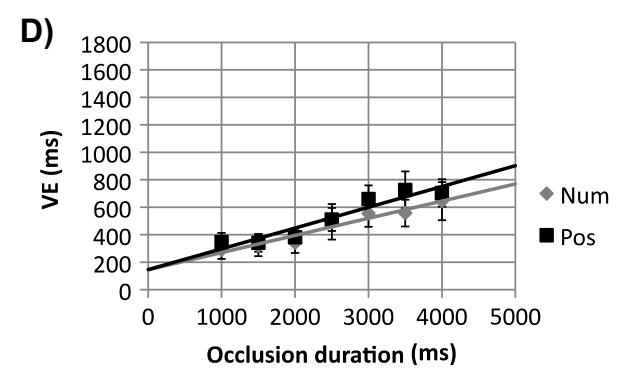

regression lines are comparable across tasks. This could be interpreted as evidence from the CRC model (Makin \& Chauhan, 2014). (Colour figure online)

multiple receptive fields can be facilitated by lateral excitatory connections. For instance, EMDs sensitive to rightward motion send excitatory signals to adjacent right-sensitive EMDs one place to the right in the EMD array. The chain of excitatory signals from one EMD to the next could continue in a straight line across occlusion. Indeed, this was demonstrated for 100 ms occlusions by Watamaniuk and McKee (1995).

Khoei, Masson, and Perrinet (2013) reported a set of computer simulations partially inspired by the trajectory network model. Based on their results and other theoretical considerations, Khoei et al. (2013) proposed that PM is mediated by a finely structured mechanisms that can be "implemented at the scale of a single cortical area" (p. 410).

Other sensory maps might have their own finely structured mechanisms which are analogous to trajectory networks. Consider the number PM task in Fig. 2b. The representation of number 10 is closely connected to the representation of 9 , then 8 , then 7 , then 6 , et cetera (Hubbard, Piazza, Pinel, \& Dehaene, 2005). After a sequence of temporally spaced number inputs, facilitator signals could be sent from one number node to the next, which then detects the input more easily, and so on. After entrainment, this chain of predictive signals could run across the occlusion period by itself (see also Supplementary Materials 1 Section 2).

To summarize, 'rate control' might be a built-in network property of all sensory systems. The correlational data provided by Makin and Bertamini (2014) and Makin and Chauhan (2014) merely showed that performance is similar across the PM tasks in Fig. 2. This does not completely rule out the SRC. 
Experiment 1 thus tested the CRC and SRC models in a novel way. Experiment 1 used position and number production tasks (like Fig. 2a-b). One group of participants were trained to respond too early on position trials in a false feedback block. They then responded earlier on both position and number trials in a subsequent probe block (where there was no feedback). Another group of participants were trained to respond too early on number trials in a false feedback block. They then responded earlier on number and position trials in the subsequent probe block. CTEs from the probe blocks are shown in Fig. 4, while the method and results are described in Supplementary Materials 3.

Experiment 1 thus suggests that position and number tasks are not cognitively independent. Instead, feedback on the position task altered CTEs on the number task, and feedback on the number task altered CTEs in the position task. These results are more consistent with the CRC than SRC.

However, the feedback effect observed in Experiment 1 might reflect the summation of many minor adjustments throughout the brain. Feedback might have altered visual velocity signals, recalibrated local rate control mechanisms, recalibrated global rate control mechanisms, introduced generic response biases, and potentiated the descending motor pathways. The feedback effect was stronger within tasks than between tasks. This suggests feedback adjusted both specific systems (used by just one task) and global systems (used by both tasks). Adjustments to the common rate control module might explain the fraction of the feedback effect that generalized across tasks. However, this claim is speculative, and we cannot completely exclude a role for generic response biases (see Supplementary materials 3 for extra analysis). Future experiments could test generalization between PM and none PM tasks to tease apart some of these explanations.

\section{Common or separate rate controllers? Summary and conclusions}

A plausible version of the SRC model proposes that trajectory networks (Watamaniuk \& McKee, 1995; Watamaniuk et al., 1995) are a built-in property in all sensory maps (Khoei et al., 2013). Prediction and updating could be implemented in a similar way across cortical regions. However, Experiment 1 found that feedback on just one kind of PM task (position or number) altered performance in two kinds of PM task (position and number). This suggests the tasks are not completely cognitively independent.

Previous work has found a sharp change in behavioural performance, brain signals, eye movements, and perception after the first 100 to $200 \mathrm{~ms}$ of occlusion (Benguigui et al., 2004; Bennett \& Barnes, 2004; Gray \& Thornton, 2001; Makin, Poliakoff, Ackerley, \& El-Deredy, 2012; Makin, Poliakoff, \& El-Deredy, 2009; Nijhawan, 1994; Sheth et al.,
2000; Tresilian, 1995). It is telling that trajectory network studies have mostly focused on automatic extrapolation across relatively short occlusions. It could be that the mechanisms described by Watamaniuk and McKee (1995) and Khoei et al. (2013) are really an account of very shortduration automatic extrapolation processes. Automatic extrapolation might also explain perceptual phenomena like visual inertia (Ramachandran \& Anstis, 1983), the flash-lag effect (Nijhawan, 1994; Sheth et al., 2000), and representational momentum (Gray \& Thornton, 2001). However, local networks may not sustain mental simulations that play out over longer periods. Indeed, sustained simulation probably requires volitional effort and does not happen automatically (Pola \& Wyatt, 1997). It is this later, effortful phase (after the first $\sim 200$ $\mathrm{ms}$ ) which could be paced by a common rate control module.

\section{No fundamental role for mental imagery or the oculomotor system in PM}

Next I aim to contrast the CRC model with other accounts that emphasize mental imagery or the oculomotor system. I believe that these accounts are flawed (albeit in interesting ways), and the common rate control model avoids these flaws.

\section{Mental imagery}

Shepard and Metzler (1971) claimed that people can mentally rotate 3-D objects using dynamic visual imagery. Following these ideas, many PM researchers have claimed that appropriate CTEs follow a period of 'mental imagery' or 'imaginary motion' (Gilden et al., 1995; Huber \& Krist, 2004; Schnider et al., 1995).

Huber and Krist (2004) carefully considered the role of mental imagery and eye movements in PM. In some conditions, participants observed a ball rolling toward the edge of a horizontal roof, and then become occluded just as it fell off. Participants pressed a button when they thought the hidden ball would hit the ground. They sometimes spontaneously tracked the hidden parabolic trajectory with their eyes. However, fixation demands had no effect on performance (unlike other studies, where fixation had small effects; Bennett, Baurès, Hecht, \& Benguigui, 2010; Makin \& Chauhan, 2014; Makin \& Poliakoff, 2011; Peterken et al., 1991). Huber and Krist (2004) thus concluded that that eye movements are epiphenomenal, and not functionally involved in PM. They claimed that internal mental imagery mediated performance.

Mental imagery was also important for de'Sperati (2003), who conceptualized eye movements as a 'precious window' into a private event that would otherwise be difficult to measure (see also Crespi, Robino, Silva, \& de'Sperati, 2012; 
A) Probe block following position trial feedback

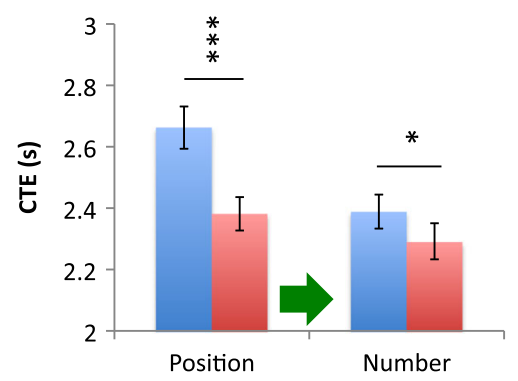

Fig. 4 Completion time estimates from position and number trials on probe blocks following feedback on just one type of task. Normal probe blocks followed a block with unbiased feedback. Adapted probe blocks followed a block with false feedback that trained participants to press too early. a After participants were trained to press too early on the position

de'Sperati \& Deubel, 2006; Jonikaitis et al., 2009). This work allows for the possibility that eye movements might facilitate or assist mental imagery, but the mental imagery is again primary.

I believe that the putative role of mental imagery in PM needs to be carefully evaluated. Unlike Pylyshyn (2003), I am not opposed the concept of mental imagery per se: After all, the CRC model claims that 'mental representations are updated during occlusion', and this is nearly synonymous with saying 'dynamic mental imagery happens during occlusion'. However, the mere occurrence of dynamic mental imagery cannot explain PM performance. The mental imagery would have to be updated at a deliberate speed. Any complete account of PM must include cognitive mechanisms which tune into the rate of change before occlusion, and then control the speed of updating during occlusion.

To give an example, Crespi et al. (2012) ran a PM task with billiard ball trajectories and argued that nonexperts used 'simulation in imagery'. But how does the brain update this imagery? There must be a mechanism which directs the imagery, and ensures that it plays out at the right speed. Ultimately, it is the director mechanism, and not just imagery itself, that is responsible for accurate performance.

\section{The oculomotor system}

Saccadic and smooth pursuit eye movements work synergistically to track moving objects (see Supplementary Materials 1 Sections 3 and 4). The oculomotor system is well understood at mechanistic and neural levels (Barnes, 2008; Lisberger, 2010). Makin and Poliakoff (2011) argued that the oculomotor system mediates position PM: Participants could track the visible targets with pursuit eye movements and continue to track as well as possible across occlusion. They would press the button when gaze reaches the end of the occluder (DeLucia \& Liddell, 1998). Even if eye movements are

\section{B) Probe block following number trial feedback}

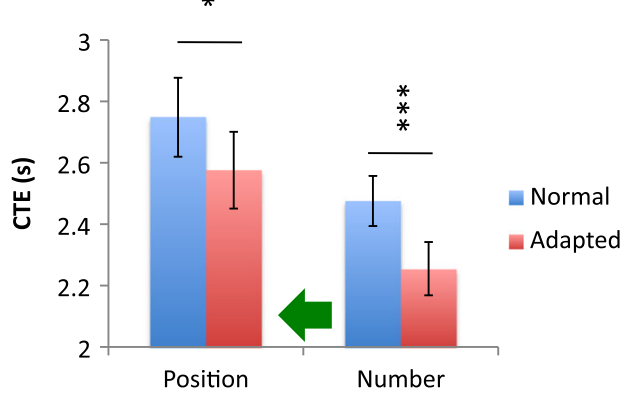

task, they started pressing earlier on the number task. b After participants were trained to press too early on the number task, they started pressing early on the position task. Green arrows show the direction of feedback generalization. Error bars $=+/-1$ SEM. $* p<.05$. *** $p<.001$. (Colour figure online)

inhibited, the oculomotor system might drive covert tracking with visuospatial attention (c.f. Rizzolatti, Riggio, Dascola, \& Umilta, 1987). Makin and Poliakoff (2011) thus proposed that the oculomotor system mediates PM during both fixation and free viewing conditions.

Several models of the pursuit system include a velocity memory component for top-down control in the absence of visual velocity signals (Barnes, 2008; Bennett \& Barnes, 2004, 2006; de Xivry, Bennett, Lefevre, \& Barnes, 2006; Lisberger, 2010). This cognitive apparatus seems well-suited for standard position PM tasks (Makin et al., 2012; Makin et al., 2008; Makin, Poliakoff, et al., 2009a; Makin, Stewart, et al., 2009b).

Many studies have found that participants can track occluded targets in an approximate fashion (e.g. Bennett \& Barnes, 2006), and that gaze position at the end of occlusion often predicts judgements (Jonikaitis et al., 2009; Makin \& Poliakoff, 2011; Wexler \& Klam, 2001). These correlations could be taken as evidence for the oculomotor account (Makin \& Poliakoff, 2011). Eye tracking during occlusion certainly indicates that the participant is cognitively engaged with the occluded motion. However, it does not demonstrate that the oculomotor system is essential for PM. Instead, I propose that the brain constructs a dynamic simulation of the occluded trajectory, and this simulation feeds both eye movements and judgments in parallel. These output channels are probably independent of each other.

Indeed, I now believe the oculomotor account in Makin and Poliakoff (2011) was limited. During position PM, the brain probably forms a continuously changing estimate of target position, which is updated at the right speed (Bosco et al., 2015). The eyes attempt to keep up with estimated target position, sometimes falling behind then jumping ahead (Bennett \& Barnes, 2006; de Xivry et al., 2006). Given such findings, we could either say (1) that the oculomotor system is guided by a dynamic representation of target position, or (2) that the oculomotor system includes apparatus that constructs 
a dynamic representation of target position. Of course, the distinction is somewhat arbitrary. However, I suspect the first formulation is superior.

When the stimulus is visible, the eyes can track high level motion gestalts that are constructed by many parts of the visual system (Barnes, 2008). Likewise, during occlusion, the eyes can track occluded acceleration (Bennett, de Xivry, Barnes, \& Lefevre, 2007) occluded curvilinear trajectories (Mrotek \& Soechting, 2007) the occluded edge of a rolling wheel (De Freitas, Myers, \& Nobre, 2016). Tracking is influenced the predictable effects of gravity and characteristic object motions learned on previous trials (Bosco, Delle Monache, \& Lacquaniti, 2012). It seems that the eyes are guided by naturalistic, time-varying representation of occluded moving objects (Bosco et al., 2015). Are the brain networks that construct these smart motion gestalts and time-varying representations part of the oculomotor system or not? This is probably stretching the definition of 'oculomotor system' to a point where the term loses meaning.

Finally, the oculomotor account cannot explain feature PM (see Fig. 2b-d). For example, the oculomotor system would simply fail to lock on to colour change or number change. If the CRC model were conclusively established by new research, we would be forced to conclude that PM is mediated by a mechanism which is not at all dedicated to oculomotor control.

\section{Summary of mental imagery and the oculomotor system}

I conclude that PM is not mediated by mental imagery (as claimed by Huber \& Krist, 2004) or by the oculomotor system (as claimed by Makin \& Poliakoff, 2011). Mental imagery may happen during PM, but the mere existence of mental imagery cannot explain performance. Meanwhile, even position PM may fundamentally depend on mechanisms outside the oculomotor network. The oculomotor system cannot function in feature space, and the putative common rate controller is certainly outside the oculomotor network.

\section{The common rate controller in interruption paradigms}

One crucial methodological distinction in the PM literature is between production tasks (see Fig. 1a), and interruption paradigms (Fig. 1b). In interruption paradigms, the target disappears and then reappears farther along its trajectory. Reappearance might be at the correct time, too early, or too late. Participants discriminate reappearance error (early or late). Crucially, there is no visible occluder in most interruption paradigms, so the exact position and time of reappearance cannot be anticipated. Again, participants could update a representation of target position at the right speed. If the target reappeared in a surprisingly advanced position, the participant would report early reappearance, but if it reappeared in a surprisingly retarded position, they would report late reappearance.

We can also run interruption paradigms in feature space. For instance, in a number interruption paradigm, the digits count down toward zero, disappear, reappear, and then continue counting down. During occlusion, the participant could mentally count at a constant rate. If the number on reappearance were surprisingly advanced, the participant would report early reappearance, if it were surprisingly retarded, they would report late reappearance.

Experiment 2 compared performance on position and number interruption paradigms. The trial structure is shown in Fig. 5. The dynamic processes reappeared after occlusion, with one of five levels of reappearance error (very early, early, on time, late, very late). Participants judged whether reappearance was too early or too late (so they were forced to give an incorrect response on the on-time trials). The proportion of 'early' reports was computed for each level of reappearance error. Supplementary Materials 4 describes the method and results.

The CRC model predicts that performance should be similar in these position and number tasks. Although there was a main effect of task, and some unexpected interactions involving speed and task, this prediction was largely confirmed (see Fig. 6a). In both tasks, participants could discriminate reappearance error successfully. Participants were also biased by speed in comparable ways (see Fig. 6b-c). Finally, sensitivity to reappearance error - the metric most closely related to the rate control function - was very similar in both tasks. Experiment 2 thus provides more evidence for CRC using the interruption paradigm rather than the production task protocol.

\section{Alternative strategies in PM}

So far, I have presented the positive case for the CRC account. For balance, the next two sections provide necessary cautions and caveats. These are very important for evaluating PM research more generally. The first problem is that PM participants can adopt multiple strategies which recruit different cognitive mechanisms.

One alternative during production tasks is called the 'clocking strategy' (DeLucia \& Liddell, 1998). The clocking strategy is to obtain a time-to-contact (TTC) estimate just before occlusion (perhaps based on an optic invariant called 'tau'; Gibson, 1979; Lee, 1976), then count this down before initiating the motor response (Tresilian, 1995). As described in Supplementary Materials 1 Section 5, some contemporary PM researchers believe the clocking strategy is used by default (Baurès et al., 2010, 2011; Bennett et al., 2010). 


\section{Position}

Numbers

First visible period (motion at 10,20 or $40 \% /$ s, first 20 to $40 \%$ of process)
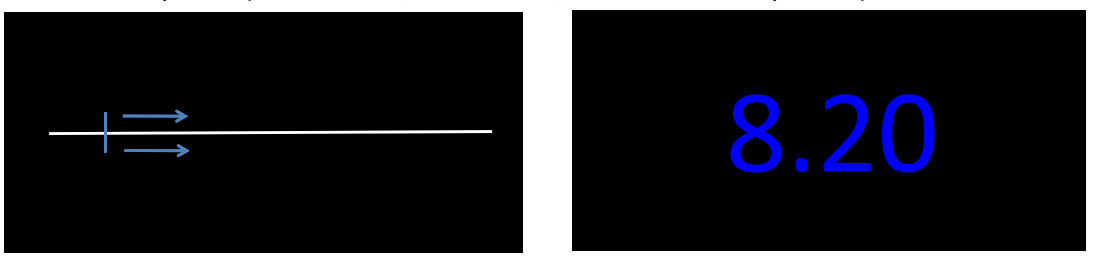

Occlusion (hidden motion 50, 25 or $0 \%$ faster or slower than in visible periods, giving 5 levels of reappearance error). The target is invisible in the Position task, the counter invisible in the Number task.
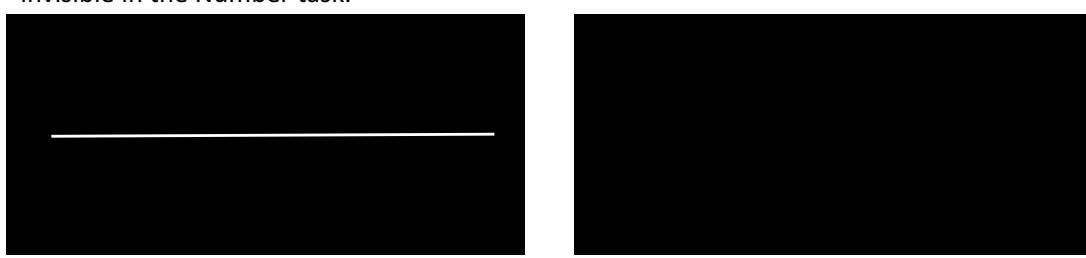

Second visible period (motion at 10,20 or $40 \% /$, last $20-40 \%$ of process)
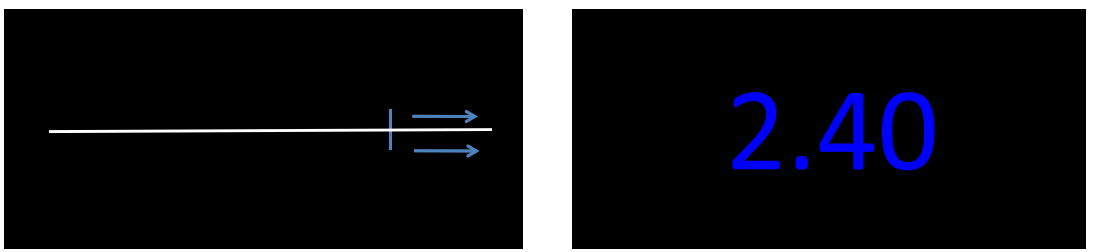

Unspeeded judgment (did the target reappear early or late?)
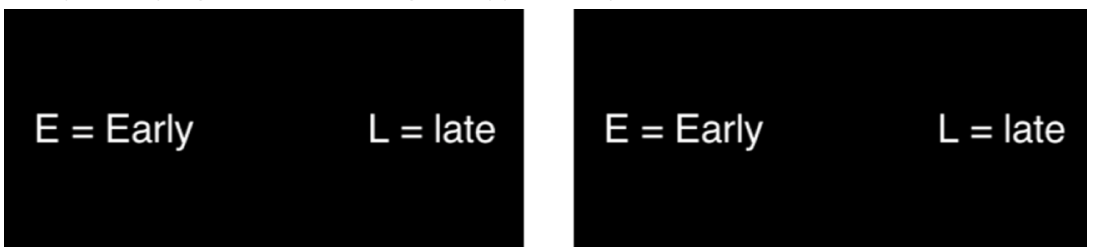

Fig. 5 Experiment 2 method

To illustrate the clocking strategy, consider a production task with a single moving target (as in Fig. 1a). TTC can be estimated at the exact point when the target reaches the occluder. The resulting TTC representation is an estimate of how long it would take for the target to reach the other side, assuming it continued at the same velocity. This TTC representation could be fed to an internal clock, which delays the motor response appropriately. If people use clocking, the occluded target position is not represented or updated, and there is no need track the target with eye movements or spatial attention. In fact, the brain could simply forget about the existence of the occluded target altogether and just run the internal clock before executing the motor response.

Participants can also use the clocking strategy in feature PM tasks. The TTC estimate could be obtained in a variety of heterogeneous ways for different kinds of feature motion, and again fed forward to the internal clock (see Fig. 7b). Again, the occluded process could be simply forgotten while the clock counts down preestimated TTC (in fact, the properties of common clock plausibly explain the VE slope similarities).

The number task requires special consideration here, because confusingly it looks like it must involve clocking. However, there is a fundamental difference between (1) mentally counting the down the numbers at the speed they were changing before occlusion and (2) using the clocking strategy, where there is no such rate-controlled simulation during occlusion. To reiterate, the key distinction is whether participants run a dynamic simulation of the occluded process (see Fig. 7a), or whether they forget about the occluded process and merely withhold a motor response for a duration equal to preestimated TTC (Fig. 7b).

It is important to recognize that clocking and rate controlled simulation are simply two available strategies. Participants could switch between the strategies arbitrarily, for example, if the cognitive load associated with one strategy feels too burdensome. It is difficult to determine whether participants use clocking or not during production tasks. 

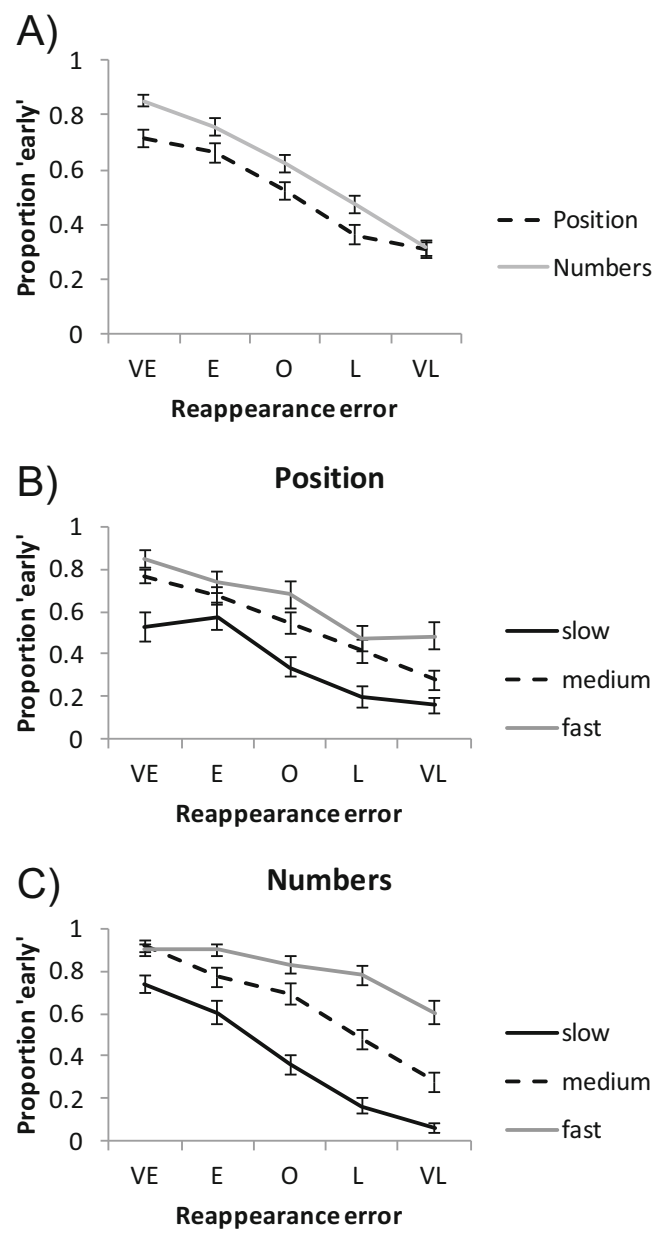

Fig. 6 a Proportion of 'early' judgements as a function of reappearance error in the position and number trials. b Data from the position task, with slow, medium, and fast trials included. $\mathbf{c}$ Data from the number task, with slow, medium, and fast trials included. Error bars $=+/-1$ SEM

DeLucia and Liddell (1998) suggest that interruption paradigms preclude clocking. At occlusion onset, the participant does not know when or where the target will reappear (there is no marked endpoint and no visible occluder). This means that participants cannot form a TTC estimate at occlusion onset. Note that if the occluder were visible, clocking would be viable in interruption paradigms.
Although DeLucia and Liddell's (1998) claim is plausible, interruption paradigms with invisible occluders can also be tackled with several 'unwanted' strategies. For instance, participants could simultaneously obtain TTC estimates for multiple locations spread along the future path of the target. At reappearance, an estimate of occlusion duration could be compared with the TTC estimate associated with that reappearance location.

However, the evidence that participants cognitively engage with the occluded target is much stronger for interruption paradigms than for production tasks. If pursuit eye movements are permitted, participants often track the targets spontaneously (Bennett \& Barnes, 2006; Makin \& Poliakoff, 2011), and eye position at reappearance reliably predicts behavioural judgments (Jonikaitis et al., 2009; Wexler \& Klam, 2001). This suggests that participants attempt to simulate ongoing motion during interruption paradigms, and the eyes are guided by the simulation. Nevertheless, alternative strategies cannot be ruled out in interruption paradigms completely. Unfortunately, there is no pure $P M$ task that can only be cracked with a single strategy.

Furthermore, DeLucia (2013) plausibly notes that PM participants might sometimes employ rough heuristics. These could even be quite explicit. In production tasks, participants might 'press promptly if the occluder looks small, but wait a long time if the occluder looks large' or 'press early if the target is going fast, but wait a long time if it is moving slowly'. In interruption paradigms, participants might compare occlusion duration to the average of all previous trials. Whether such heuristics produce good performance or not depends on how well spatial and temporal confounds are controlled (as described in Supplementary Materials 2 and 4).

These cautions are sobering for PM researchers. The best we can do is replicate theoretically interesting effects with different response protocols. Previous work found a theoretically interesting similarity between position and number tasks using the production task protocol (where clocking is viable). Experiment 2 replicated this similarity with an interruption
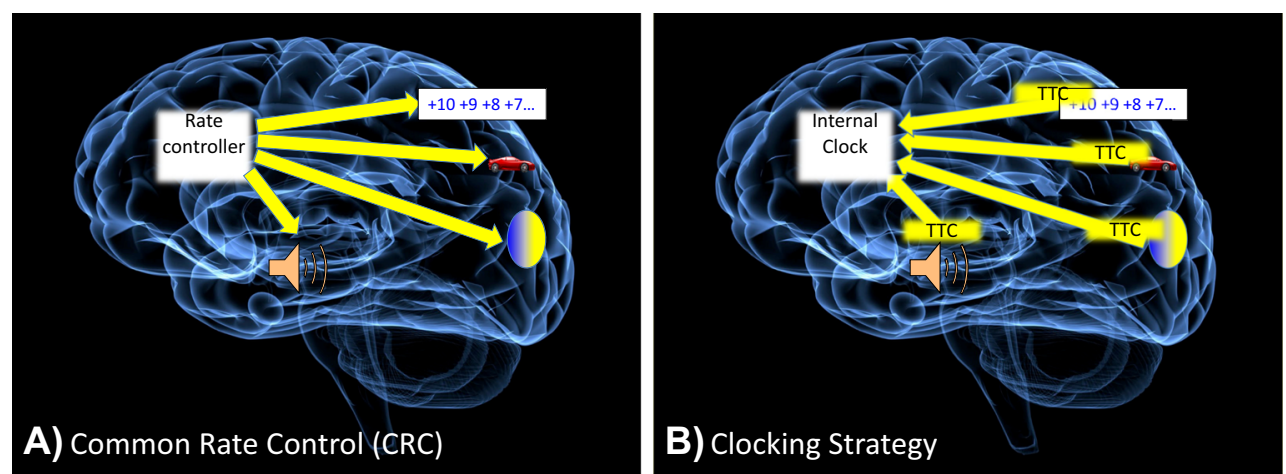

Fig. 7 Cartoon depictions of (a) the common rate control (CRC) and (b) the clocking strategy. (Colour figure online) 
paradigm (where clocking is not viable without modification, and is less likely). We can thus tentatively conclude that the position-number similarity arises from a common rate controller (see Fig. 7a) rather than a common clocking stage (Fig. 7b).

\section{Alternative explanations for PM effects}

We can now move on to another issue with PM research. Many observed effects can be explained by changes to subjective velocity before occlusion (Bennett et al., 2010). For example, if the target looks slow, participants will press later; if it looks fast, they will press earlier. Any factor which influences perceived velocity during the visible period must influence CTEs (whatever strategy participants are using). For example, moving objects often appear faster if fixation is required, and this could be why CTEs are often shorter during fixation (Makin \& Poliakoff, 2011).

Several PM experiments have documented dual-task interference costs (Baurès, Bennett, \& Causer, 2015; Baurès, DeLucia, Olson, \& Oberfeld, 2017; Baurès et al., 2010, 2011; DeLucia \& Novak, 1997; Lyon \& Waag, 1995; Oberfeld \& Hecht, 2008). These experiments illustrate the difference between pre and post occlusion explanations. For example, Baurès et al. (2010) presented two PM tasks concurrently (with one horizontal path slightly above the other). Participants made one CTE of the first-arriving target and then a second CTE for the second-arriving target. CTEs for the first-arriving target were equivalent to CTEs measured in a one-object baseline condition. However, CTEs for the second-arriving target were delayed compared to the oneobject baseline condition. The dual-task interference was thus asymmetrical, with all costs falling on the second CTE. In a follow up study, Baurès et al. (2011) observed the same asymmetrical interference effect when a cue at occlusion onset indicated that one of the two targets could be ignored. Therefore, the interference must have happened in the visible period, before occlusion.

The SRC model suggests two PM tasks could run in parallel, without interference. Perhaps the dual-task interference found by Baurès et al. (2010) supports the CRC model? However, the dual-task interference might arise from a visual bottleneck that limits motion processing before occlusion (Baurès et al., 2011), so it does not provide definitive evidence for the CRC model (although interference between concurrent visual and auditory PM tasks might be instructive in future work).

In summary, any manipulation which distorts subjective velocity before occlusion (such as fixation commands or secondary tasks) is likely to shift PM performance accordingly. This is true whether people use the clocking strategy or run rate-controlled simulations.

\section{So, what is the 'common rate controller' anyway?}

Many researchers believe there is a 'core timing system' in the brain (Coull, Cheng, \& Meck, 2011). We can thus ask whether the rate controller can be reduced to this core timing system. Contemporary PM papers rarely make these links, so it is worth working through these speculative possibilities in detail.

\section{What is the core timing system?}

The classic pacemaker-accumulator clock model provides a framework for understanding time perception and timed behaviour in humans and animals (Coull et al., 2011; Wearden, 2013). A simplified diagram of the pacemaker-accumulator clock model is shown in Fig. 8a. The pacemaker emits ticks at regular intervals, when the switch closes, and the ticks pass into the accumulator. The final value in the accumulator is committed to memory and represents subjective duration of the stimulus.

Some researchers claim this internal clock is part of a core timing system, centred on the basal ganglia (BG), particularly the dorsal striatum, and pre-supplementary motor areas (preSMA). These brain regions have been repeatedly identified in fMRI studies of interval timing (Coull, Vidal, Nazarian, \& Macar, 2004; Lewis \& Miall, 2003). Indeed, after reviewing fMRI work, Coull et al. (2011) concluded that 'results are suggestive of a centralized, context independent, supramodal timer localized in the dorsal striatum of the $\mathrm{BG}^{\prime}$ (p. 7). The vital role of the $\mathrm{BG}$ in timing has also been demonstrated by lesion and pharmacological studies in animals.

\section{Is the common rate controller the same thing as the core timing system?}

I tentatively propose that this 'centralized, supramodal timer' incorporating the BG could mediate the rate control function (see Fig. 8c). The pacemaker could be temporarily coupled to representations of the occluded process in PM tasks, while it could be coupled to an accumulator in other interval timing tasks. Prior to occlusion, the temporary network could become sensitive to how much change happens per tick of the pacemaker. Then, during occlusion, the pacemaker could continue ticking and drive mental updating at approximately the same rate. Local predictive mechanisms within each sensory map could still be present, but these may cover very short occlusions only. This model was also outlined in Makin (2017) and is clearly distinct from how the internal clock would be deployed if people use the clocking strategy (see Fig. 8b). 


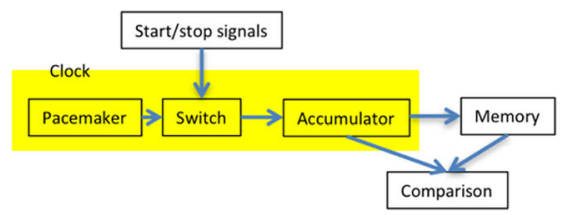

A) Pacemaker- accumulator clock model

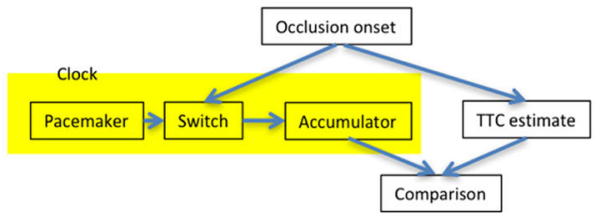

B) How the pacemaker-accumulator clock might work if participants used a clocking strategy

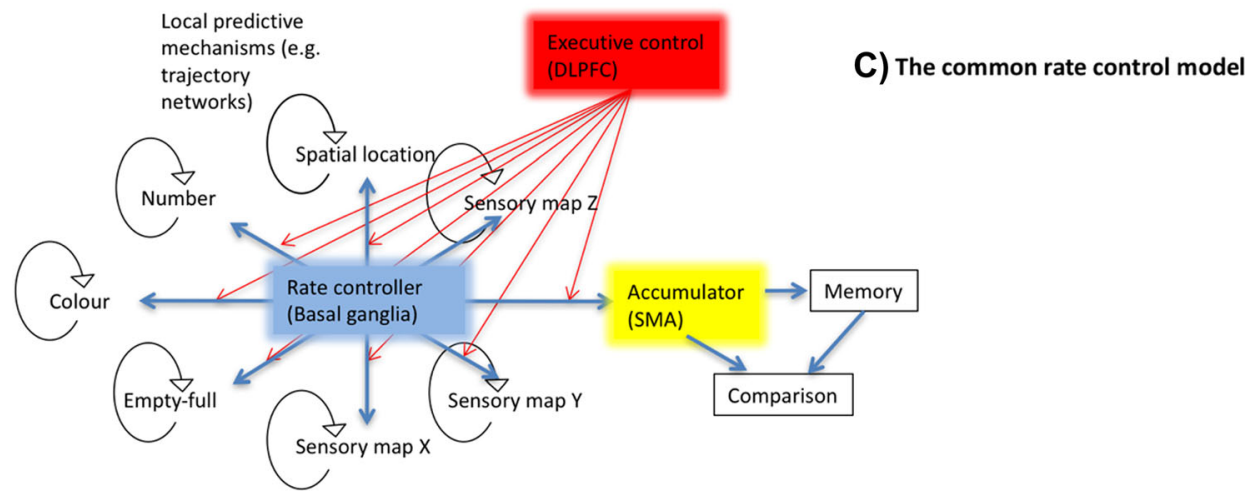

Fig. 8 Relationships between CRC and the internal clock model. a Basic schematic of the pacemaker-accumulator clock model. b How this internal clock could be recruited if participants used a clocking strategy in PM tasks. c Speculative version of the common rate control model. The pacemaker/rate controller component could be functionally coupled to sensory maps and guide updating during occlusion. The accumulator could be just one target module of the pacemaker/rate controller. The pacemaker/rate controller could be implemented by basal ganglia circuitry. The DLPFC could have an executive role, switching connections between the rate controller and other modules. Local extrapolation probably occurs within the sensory maps (curved arrows) but the rate controller could be recruited during long occlusions. (Colour figure online)
Some researchers disagree with the core timing system hypothesis and believe that temporal information is distributed throughout the cortex (e.g. Johnston, Arnold, \& Nishida, 2006). However, evidence for a single supramodal clock and distributed timing can be reconciled. Coull et al. (2011) suggest that distributed timing covers the subsecond range, while there could still be a supramodal clock which is recruited for longer intervals (Coull et al., 2011; Lewis \& Miall, 2003). This fits well with the claim that the $\mathrm{CRC}$ is only recruited during longer occlusions.

\section{Neuroimaging evidence that the common rate controller is related to the core timing system}

Using fMRI, Lencer et al. (2004) compared brain activations from visible and occluded tracking conditions. Participants attempted to follow all targets with eye movements. The DLPFC was selectively activated in the occlusion condition. Makin and Chauhan (2014) thus proposed that the DLPFC mediates rate control. However, Lencer et al. (2004) found several other activations during occlusion, including subregions of the cerebellum, the supplementary and pre-supplementary eye fields, and intriguingly, the basal ganglia and premotor cortex (that is, the centre of the core timing system). As Lencer et al. (2004) pointed out, increased saccade frequency could explain some of the occlusion-related activations. However converging evidence comes from O'Driscoll et al. (2000), who found that the caudate nucleus of the BG was activated during predictive pursuit of sinusoidal target motion.

These studies support the notion that the BG circuitry functions like a central rate controller, which can be used to control simulations of target motion. The same rate controller could control simulations of motion in feature space. Meanwhile, the DLPFC could have an executive role, or inhibit irrelevant and disruptive visual inputs during occlusion. In Fig. 8c, the DLPFC is envisaged as providing a command signal, facilitating coupling between the rate controller and cortical sensory maps, or between the rate controller and the accumulator.

There is also some fMRI evidence that the cerebellum mediates PM (O'Reilly et al., 2008). However, brain lesion studies suggests the cerebellum is not essential for performance but may fine-tune performance based on feedback (Deluca et al., 2014; Roth et al., 2013). Therefore, the cerebellum is probably not the neural home of the rate controller (see Supplementary Materials 1 Sections 6-8). 


\section{Dissociation between short and long occlusions}

It is worth reemphasising that PM researchers should not expect the same brain mechanisms to mediate PM tasks with shorter and longer occlusions (although the frontier line dividing 'short' from 'long' is fuzzy). Various predictive mechanisms seem to cover 100-200 ms of occlusion automatically (Benguigui et al., 2004; Bennett \& Barnes, 2006; Tresilian, 1995). These mechanisms overcome visuomotor delay during smooth pursuit and may explain flash lag effect (Nijhawan, 1994) and representational momentum (Gray \& Thornton, 2001). The CRC may be solely responsible for updating after these local predictive mechanisms fade. This may happen at slightly different times in different PM tasks. When designing experiments to probe CRC function, occlusions of around 1 second or longer are advisable.

\section{Future PM experiments}

Given existing neuroscientific evidence, one could plausibly associate the putative rate controller with one of many brain regions, including the modules of the frontoparietal attentional system, the DLPFC, core timing networks in the dorsal striatum of the BG and pre-SMA, or perhaps the cerebellum. These could all be regions of interest in future fMRI studies. More interestingly, we could use fMRI to falsify the CRC model: If position, number, colour, and accumulation PM tasks produce totally different patterns of brain activity, with no common node, the CRC model would have to be reconsidered. However, I predict that this common node would only be reliably activated when occlusion duration exceeds 1 second (and certainly more than $200 \mathrm{~ms}$ ).

\section{Conclusions}

The prediction-motion literature is fragmented. However, we can nevertheless distil four novel claims, which can serve as the foundation for future research:

1. Local predictive circuits can cover short occlusions. However, a common rate controller is recruited for longer occlusions.

2. The common rate control model explains more PM data than mental imagery or oculomotor accounts.

3. Unfortunately, there is no pure PM task that uniquely taps a single cognitive mechanism. However, observed intertask similarities probably arise from a common rate controller.

4. The rate controller might be reduced to the core timing system, incorporating the basal ganglia.
Acknowledgements A Leverhulme Trust Early Career Fellowship (ECF-2012-721) funded Alexis Makin.

Open Access This article is distributed under the terms of the Creative Commons Attribution 4.0 International License (http:// creativecommons.org/licenses/by/4.0/), which permits unrestricted use, distribution, and reproduction in any medium, provided you give appropriate credit to the original author(s) and the source, provide a link to the Creative Commons license, and indicate if changes were made.

\section{References}

Alink, A., Schwiedrzik, C. M., Kohler, A., Singer, W., \& Muckli, L. (2010). Stimulus predictability reduces responses in primary visual cortex. Journal of Neuroscience, 30(8), 2960-2966. https://doi.org/ 10.1523/jneurosci.3730-10.2010

Barnes, G. R. (2008). Cognitive processes involved in smooth pursuit eye movements. Brain and Cognition, 68(3), 309-326. https://doi.org/ 10.1016/j.bandc.2008.08.020

Battaglini, L., Campana, G., \& Casco, C. (2013). Illusory speed is retained in memory during invisible motion. I-Perception, 4(3), 180-191. https://doi.org/10.1068/i0562

Baurès, R., Bennett, S. J., \& Causer, J. (2015). Temporal estimation with two moving objects: Overt and covert pursuit. Experimental Brain Research, 233(1), 253-261. https://doi.org/10.1007/s00221-0144110-y

Baurès, R., DeLucia, P. R., Olson, M., \& Oberfeld, D. (2017). Asymmetric interference in concurrent time-to-contact estimation: Cousin or twin of the psychological refractory period effect? Attention, Perception, \& Psychophysics, 79(2), 698-711. https:// doi.org/10.3758/s13414-016-1244-y

Baurès, R., Oberfeld, D., \& Hecht, H. (2010). Judging the contact-times of multiple objects: Evidence for asymmetric interference. Acta Psychologica, 134(3), 363-371. https://doi.org/10.1016/j.actpsy. 2010.03.009

Baurès, R., Oberfeld, D., \& Hecht, H. (2011). Temporal-range estimation of multiple objects: Evidence for an early bottleneck. Acta Psychologica, 137(1), 76-82. https://doi.org/10.1016/j.actpsy. 2011.03.002

Becker, W., \& Fuchs, A. F. (1985). Prediction in the oculomotor system-Smooth pursuit during transient disappearance of a visual target. Experimental Brain Research, 57(3), 562-575.

Benguigui, N., Broderick, M., \& Ripoll, H. (2004). Age differences in estimating arrival-time. Neuroscience Letters, 369(3), 197-202. https://doi.org/10.1016/j.neulet.2004.07.051

Bennett, S. J., \& Barnes, G. R. (2003). Human ocular pursuit during the transient disappearance of a visual target. Journal of Neurophysiology, 90(4), 2504-2520. https://doi.org/10.1152/jn. 01145.2002

Bennett, S. J., \& Barnes, G. R. (2004). Predictive smooth ocular pursuit during the transient disappearance of a visual target. Journal of Neurophysiology, 92(1), 578-590. https://doi.org/10.1152/jn. 01188.2003

Bennett, S. J., \& Barnes, G. R. (2006). Combined smooth and saccadic ocular pursuit during the transient occlusion of a moving visual object. Experimental Brain Research, 168(3), 313-321. https://doi. org/10.1007/s00221-005-0101-3

Bennett, S. J., Baurès, R., Hecht, H., \& Benguigui, N. (2010). Eye movements influence estimation of time-to-contact in prediction motion. Experimental Brain Research, 206(4), 399-407. https://doi.org/10. 1007/s00221-010-2416-y 
Bennett, S. J., \& Benguigui, N. (2013). Is acceleration used for ocular pursuit and spatial estimation during prediction motion? PLOS ONE, 8(5), e63382. https://doi.org/10.1371/journal.pone.0063382

Bennett, S. J., de Xivry, J. J. O., Barnes, G. R., \& Lefevre, P. (2007). Target acceleration can be extracted and represented within the predictive drive to ocular pursuit. Journal of Neurophysiology, 98(3), 1405-1414. https://doi.org/10.1152/jn.00132.2007

Blaser, E., Pylyshyn, Z. W., \& Holcombe, A. O. (2000). Tracking an object through feature space. Nature, 408(6809), 196-199. https:// doi.org/10.1038/35041567

Blaser, E., \& Sperling, G. (2008). When is motion "motion"? Perception, 37(4), 624-627. https://doi.org/10.1068/p5812

Bosco, G., Delle Monache, S., \& Lacquaniti, F. (2012). Catching what we can't see: Manual Interception of occluded fly-ball trajectories. PLOS ONE, 7(11). doi:e49381 https://doi.org/10.1371/journal. pone. 0049381

Bosco, G., Monache, S. D., Gravano, S., Indovina, I., La Scaleia, B., Maffei, V., . . . Lacquaniti, F. (2015). Filling gaps in visual motion for target capture. Frontiers in Integrative Neuroscience, 9(13), 117. https://doi.org/10.3389/fnint.2015.00013

Burr, D., \& Thompson, P. (2011). Motion psychophysics: 1985 to 2010. Vision Research, 51(13), 1431-1456. https://doi.org/10.1016/j. visres.2011.02.008

Churchland, M. M., Chou, I. H., \& Lisberger, S. G. (2003). Evidence for object permanence in the smooth-pursuit eye movements of monkeys. Journal of Neurophysiology, 90(4), 2205-2218.

Coull, J. T., Cheng, R. K., \& Meck, W. H. (2011). Neuroanatomical and neurochemical substrates of timing. Neuropsychopharmacology, 36(1), 3-25. https://doi.org/10.1038/npp.2010.113

Coull, J. T., Vidal, F., Nazarian, B., \& Macar, F. (2004). Functional anatomy of the attentional modulation of time estimation. Science, 303(5663), 1506-1508. https://doi.org/10.1126/science.1091573

Crespi, S., Robino, C., Silva, O., \& de'Sperati, C. (2012). Spotting expertise in the eyes: Billiards knowledge as revealed by gaze shifts in a dynamic visual prediction task. Journal of Vision, 12(11). https:// doi.org/10.1167/12.11.30

de'sperati, C. (2003). Precise oculomotor correlates of visuospatial mental rotation and circular motion imagery. Journal of Cognitive Neuroscience, 15(8), 1244-1259. https://doi.org/10.1162/ 089892903322598184

de'sperati, C., \& Deubel, H. (2006). Mental extrapolation of motion modulates responsiveness to visual stimuli. Vision Research, 46(16), 2593-2601. https://doi.org/10.1016/j.visres.2005.12.019

De Freitas, J., Myers, N. E., \& Nobre, A. C. (2016). Tracking the changing feature of a moving object. Journal of Vision, 16(3), 1-21. https://doi.org/10.1167/16.3.22

de Xivry, J. J. O., Bennett, S. J., Lefevre, P., \& Barnes, G. R. (2006). Evidence for synergy between saccades and smooth pursuit during transient target disappearance. Journal of Neurophysiology, 95(1), $418-427$.

Deluca, C., Golzar, A., Santandrea, E., Lo Gerfo, E., Estocinova, J., Moretto, G., . . Chelazzi, L. (2014). The cerebellum and visual perceptual learning: Evidence from a motion extrapolation task. Cortex, 58, 52-71. https://doi.org/10.1016/j.cortex.2014.04.017

DeLucia, P. R. (2013). Effects of size on collision perception and implications for perceptual theory and transportation safety. Current Directions in Psychological Science, 22, 199-204. https://doi.org/ 10.1177/0963721412471679

DeLucia, P. R., \& Liddell, G. W. (1998). Cognitive motion extrapolation and cognitive clocking in prediction motion tasks. Journal of Experimental Psychology: Human Perception and Performance, 24(3), 901-914. https://doi.org/10.1037/0096-1523.24.3.901

DeLucia, P. R., \& Novak, J. B. (1997). Judgments of relative time-tocontact of more than two approaching objects: Toward a method. Perception \& Psychophysics, 59(6), 913-928. https://doi.org/10. 3758/BF03205508
DeLucia, P. R., Tresilian, J. R., \& Meyer, L. E. (2000). Geometrical illusions can affect time-to-contact estimation and mimed prehension. Journal of Experimental Psychology: Human Perception and Performance, 26(2), 552-567. https://doi.org/10.1037/0096-1523.26.2.552

Diwakar, M., Harrington, D. L., Maruta, J., Ghajar, J., El-Gabalawy, F., Muzzatti, L., . . . Lee, R. R. (2015). Filling in the gaps: Anticipatory control of eye movements in chronic mild traumatic brain injury. NeuroImage: Clinical, 8, 210-223. https://doi.org/10.1016/j.nicl. 2015.04.011

Gibson, J. J. (1979). The ecological approach to visual perception. Hillsdale, NJ: Erlbaum.

Gilden, D., Blake, R., \& Hurst, G. (1995). Neural adaptation of imaginary visual-motion. Cognitive Psychology, 28(1), 1-16. https://oi.org/ 10.1006/cogp.1995.1001

Gottsdanker, R. M. (1956). The ability of human operators to detect acceleration of target motion. Psychological Bulletin, 53, 477-487.

Gray, R., \& Thornton, I. M. (2001). Exploring the link between time to collision and representational momentum. Perception, 30(8), 10071022.

Hancock, P. A., \& Manser, M. P. (1997). Time-to-contact: More than tau alone. Ecological Psychology, 9(4), 265-297. https://doi.org/10. 1207/s15326969eco0904 2

Hecht, H., \& Savelsburgh, G. (2004). Theories of time-to-contact judgment. In H. Hecht \& G. Savelsburgh (Eds.), Time-to-contact (pp. 111). Amsterdam, Netherlands: Elsevier.

Hooker, C., \& Park, S. (2000). Trajectory estimation in schizophrenia. Schizophrenia Research, 45(1/2), 83-92.

Horswill, M. S., Helman, S., Ardiles, P., \& Wann, J. P. (2005). Motorcycle accident risk could be inflated by a time to arrival illusion. Optometry and Vision Science, 82(8), 740-746. https://doi.org/ 10.1097/01.opx.0000175563.21423.50

Hubbard, E. M., Piazza, M., Pinel, P., \& Dehaene, S. (2005). Interactions between number and space in parietal cortex. Nature Reviews Neuroscience, 6(6), 435-448. https://doi.org/10.1038/nrn1684

Huber, S., \& Krist, H. (2004). When is the ball going to hit the ground? Duration estimates, eye movements, and mental imagery of object motion. Journal of Experimental Psychology: Human Perception and Performance, 30(3), 431-444. https://doi.org/10.1037/00961523.30.3.431

Johnston, A., Arnold, D. H., \& Nishida, S. (2006). Spatially localized distortions of event time. Current Biology, 16(5), 472-479. https:// doi.org/10.1016/j.cub.2006.01.032

Jonikaitis, D., Deubel, H., \& de'Sperati, C. (2009). Time gaps in mental imagery introduced by competing saccadic tasks. Vision Research, 49(17), 2164-2175. https://doi.org/10.1016/j.visres.2009.05.021

Khoei, M. A., Masson, G. S., \& Perrinet, L. U. (2013). Motion-based prediction explains the role of tracking in motion extrapolation. Journal of Physiology-Paris, 107(5), 409-420. https://doi.org/10. 1016/j.jphysparis.2013.08.001

Lee, D. N. (1976). Theory of visual control of braking based on information about time-to-collision. Perception, 5(4), 437-459. https://doi. org/10.1068/p050437

Lencer, R., Nagel, M., Sprenger, A., Zapf, S., Erdmann, C., Heide, W., \& Binkofski, F. (2004). Cortical mechanisms of smooth pursuit eye movements with target blanking: An fMRI study. European Journal of Neuroscience, 19(5), 1430-1436.

Lewis, P. A., \& Miall, R. C. (2003). Distinct systems for automatic and cognitively controlled time measurement: evidence from neuroimaging. Current Opinion in Neurobiology, 13(2), 250-255. https:// doi.org/10.1016/s0959-4388(03)00036-9

Lisberger, S. G. (2010). Visual guidance of smooth-pursuit eye movements: Sensation, action, and what happens in between. Neuron, 66(4), 477-491. https://doi.org/10.1016/j.neuron.2010.03.027

Lugtigheid, A. J., \& Welchman, A. E. (2011). Evaluating methods to measure time-to-contact. Vision Research, 51(20), 2234-2241. https://doi.org/10.1016/j.visres.2011.08.019 
Lyon, D. R., \& Waag, W. L. (1995). Time-course of visual extrapolation accuracy. Acta Psychologica, 89(3), 239-260. https://doi.org/10. 1016/0001-6918(95)98945-Z

Makin, A. D. J. (2017). Choosing the speed of dynamic mental simulations. In Progress in brain research. https://doi.org/10.1016/bs.pbr. 2017.05.001

Makin, A. D. J., \& Bertamini, M. (2014). Do different types of dynamic extrapolation rely on the same mechanism? Journal of Experimental Psychology: Human Perception and Performance, 40(4), 15661579. https://doi.org/10.1037/a0036680.

Makin, A. D. J., \& Chauhan, T. (2014). Memory-guided tracking through physical space and feature space. Journal of Vision, 14(13). https:// doi.org/10.1167/14.13.10

Makin, A. D. J., \& Poliakoff, E. (2011). Do common systems control eye movements and motion extrapolation? Quarterly Journal of Experimental Psychology, 64(7), 1327-1343. https://doi.org/10. 1080/17470218.2010.548562

Makin, A. D. J., Poliakoff, E., Ackerley, R., \& El-Deredy, W. (2012). Covert tracking: A combined ERP and fixational eye movement study. PLOS ONE, 7(6), e38479. https://doi.org/10.1371/journal. pone.0038479

Makin, A. D. J., Poliakoff, E., Chen, J., \& Stewart, A. J. (2008). The effect of previously viewed velocities on motion extrapolation. Vision Research, 48(18), 1884-1893. https://doi.org/10.1016/j. visres.2008.05.023

Makin, A. D. J., Poliakoff, E., \& El-Deredy, W. (2009a). Tracking visible and occluded targets: Changes in event related potentials during motion extrapolation. Neuropsychologia, 47(4), 1128-1137. https://doi.org/10.1016/j.neuropsychologia.2009.01.010

Makin, A. D. J., Stewart, A. J., \& Poliakoff, E. (2009b). Typical object velocity influences motion extrapolation. Experimental Brain Research, 193(1), 137-142. https://doi.org/10.1007/s00221-0081678-0

Mrotek, L. A., \& Soechting, J. F. (2007). Predicting curvilinear target motion through an occlusion. Experimental Brain Research, 178(1), 99-114. https://doi.org/10.1007/s00221-006-0717-y

Nagel, M., Sprenger, A., Nitschke, M., Zapf, S., Heide, W., Binkofski, F., \& Lencer, R. (2007). Different extraretinal neuronal mechanisms of smooth pursuit eye movements in schizophrenia: An fMRI study. NeuroImage, 34(1), 300-309. https:doi.org/10.1016/j.neuroimage. 2006.08.025

Nijhawan, R. (1994). Motion extrapolation in catching. Nature, 370(6487), 256-257. https://doi.org/10.1038/370256b0

O’Driscoll, G. A., Wolff, A. L. V, Benkelfat, C., Florencio, P. S., Lal, S., \& Evans, A. C. (2000). Functional neuroanatomy of smooth pursuit and predictive saccades. NeuroReport, 11(6), 1335-1340. https:// doi.org/10.1097/00001756-200004270-00037

O'Reilly, J. X., Mesulam, M. M., \& Nobre, A. C. (2008). The cerebellum predicts the timing of perceptual events. Journal of Neuroscience, 28(9), 2252-2260. https://doi.org/10.1523/jneurosci.2742-07.2008

Oberfeld, D., \& Hecht, H. (2008). Effects of a moving distractor object on time-to-contact judgments. Journal of Experimental Psychology: Human Perception and Performance, 34(3), 605-623.

Peterken, C., Brown, B., \& Bowman, K. (1991). Predicting the future position of a moving target. Perception, 20(1), 5-16. https://doi.org/ $10.1068 / \mathrm{p} 200005$

Pola, J., \& Wyatt, H. J. (1997). Offset dynamics of human smooth pursuit eye movements: Effects of target presence and subject attention. Vision Research, 37(18), 2579-2595. https://doi.org/10.1016/ S0042-6989(97)00058-8

Pylyshyn, Z. W. (2003). Mental imagery: In search of a theory. Behavioral and Brain Sciences, 25(2), 157-238. https://doi.org/10. 1017/S0140525X02000043

Ramachandran, V. S., \& Anstis, S. M. (1983). Extrapolation of motion path in human visual perception. Vision Research, 23(1), 83-85. https://doi.org/10.1016/0042-6989(83)90044-5
Rizzolatti, G., Riggio, L., Dascola, I., \& Umilta, C. (1987). Reorienting attention across the horizontal and vertical meridians-Evidence in favour of a premotor theory of attention. Neuropsychologia, 25(1A), 31-40. https://doi.org/10.1016/0028-3932(87)90041-8

Rosenbaum, D. A. (1975). Perception and extrapolation of velocity and acceleration. Journal of Experimental Psychology: Human Perception and Performance, 1(4), 395-403. https://doi.org/10. 1037/0096-1523.1.4.395

Roth, M. J., Synofzik, M., \& Lindner, A. (2013). The cerebellum optimizes perceptual predictions about external sensory events. Current Biology, 23(10), 930-935. https://doi.org/10.1016/j.cub.2013.04.027

Schiff, W., \& Detwiler, M. L. (1979). Information used in judging impending collision. Perception, 8(6), 647-658. https://doi.org/10. 1068/p080647

Schiff, W., \& Oldak, R. (1990). Accuracy of judging time to arrival: Effects of modality, trajectory, and gender. Journal of Experimental Psychology: Human Perception and Performance, 16(2), 303-316. https://doi.org/10.1037/0096-1523.16.2.303

Schnider, A., Gutbrod, K., \& Hess, C. W. (1995). Motion imagery in Parkinson's disease. Brain, 118, 485-493. https://doi.org/10.1093/ brain/118.2.485

Shepard, R. N., \& Metzler, J. (1971). Mental rotation of 3-dimensional objects. Science, 171(3972), 701-703. https://doi.org/10.1126/ science.171.3972.701

Sheth, B. R., Nijhawan, R., \& Shimojo, S. (2000). Changing objects lead briefly flashed ones. Nature Neuroscience, 3(5), 489-495. https:// doi.org/10.1038/74865

Slater-Hammel, A. T. (1955). Estimation of movement as a function of the distance of movement perception and target distance. Perception and Motor Skills, 5, 201-204. https://doi.org/10.2466/pms.1955.5.3.201

Sokolov, A., \& Pavlova, M. (2003). Timing accuracy in motion extrapolation: Reverse effects of target size and visible extent of motion at low and high speeds. Perception, 32(6), 699-706. https://doi.org/10. 1068/p3397

Travis, R. C., \& Dodge, R. (1930). Ocular pursuit of objects which temporarily disappear. Journal of Experimental Psychology, 13(1), 98112. https://doi.org/10.1037/h0074637

Tresilian, J. R. (1995). Perceptual and cognitive-processes in time-tocontact estimation: Analysis of prediction-motion and relative judgment tasks. Perception \& Psychophysics, 57(2), 231-245. https:// doi.org/10.3758/BF03206510

Vagnoni, E., Lourenco, S. F., \& Longo, M. R. (2012). Threat modulates perception of looming visual stimuli. Current Biology, 22(19), 826827. https://doi.org/10.1016/j.cub.2012.07.053

von Hofsten, C., Kochukhova, O., \& Rosander, K. (2007). Predictive tracking over occlusions by 4 -month-old infants. Developmental Science, 10(5), 625-640. https://doi.org/10.1111/j.1467-7687.2007. 00604.x

Watamaniuk, S. N. J., \& McKee, S. P. (1995). Seeing motion behind occluders. Nature, 377(6551), 729-730. https://doi.org/10.1038/ $377729 \mathrm{a} 0$

Watamaniuk, S. N. J., McKee, S. P., \& Grzywacz, N. M. (1995). Detecting a trajectory embedded in random-direction motion noise. Vision Research, 35(1), 65-77. https://doi.org/10.1016/00426989(94)e0047-o

Wearden, J. H. (2013). The cognitive neuroscience of time perception: How psychological studies might help to dissect the timing system. Neuropsychologia, 51(2), 187-190. https://doi.org/10.1016/j. neuropsychologia.2012.09.035

Wexler, M., \& Klam, F. (2001). Movement prediction and movement production. Journal of Experimental Psychology: Human Perception and Performance, 27(1), 48-64. https://doi.org/10. 1037/0096-1523.27.1.48

Wiener, E. L. (1962). Motion prediction as a function of target speed and duration of presentation. Journal of Applied Psychology, 46(6), 420-424. https://doi.org/10.1037/h0041224 